\title{
Indifference Pricing of Reinsurance with Reinstatements Using Coherent Monetary Criteria
}

\author{
Nabil KAZI-TANI*
}

December 15, 2020

\begin{abstract}
We consider the problem of indifference pricing of reinsurance contracts that contain a reinstatement clause. We define the indifference price relative to both a monetary utility function and a risk measure, to take into account both the risk reduction and the relief of capital immobilization provided by reinsurance. We characterize the indifference price as the unique solution to a fixed point equation and we bound the price by two easily computable values, if one has access to losses simulations. We illustrate our results on a European catastrophe insurance portfolio, and we conduct a simulation study for comparison and reproducibility purposes, where we include the case of dependence between claim arrivals, using Hawkes processes.
\end{abstract}

Key words: Insurance premium calculation, Convex risk measures, Monetary utility functions, Reinsurance layers, Reinstatements.

AMS 2020 subject classifications: 91G05, 91B05, 91B16, $62 \mathrm{P} 05$.

\section{Contents}

1 Introduction 2

1.1 Summary of the Approach . . . . . . . . . . . . . . . . . 2

1.2 Related Literature . . . . . . . . . . . . . . . . . . . . . . . . 3

2 Model and Assumptions 3

2.1 Monetary Risk Measures and Utility Functions . . . . . . . . . . . . . . . 3

2.2 Non Linear Contract with Reinstatements . . . . . . . . . . . . . . . . . 5

3 Indifference Pricing and Bounds $\quad \mathbf{7}$

3.1 Cost of Capital and Cash Super-additivity . . . . . . . . . . . . . 8

3.2 A Fixed Point and Pricing Bounds . . . . . . . . . . . . . . . . . 9

3.3 Asymptotic Analysis . . . . . . . . . . . . . . . . . . . . . . . 13

4 Numerical Analysis and Examples $\quad 14$

4.1 Results for a Real Insurance Portfolio . . . . . . . . . . . . . . . . . . . . . . . . 14

4.2 Simulated Losses and Hawkes Processes . . . . . . . . . . . . . . . . . . . 15

${ }^{*}$ Univ Lyon, Université de Lyon 1, Laboratoire de Sciences Actuarielle et Financière, ISFA, 50 Avenue Tony Garnier, F-69007 Lyon, France. nabil.kazi-tani@univ-lyon1.fr.

The author gratefully acknowledges the support of the insurer Axa who made its data available, and thanks Thierry Cohignac, Guillaume Gorge and Jean-Sébastien Lagacé for fruitful discussions. 


\section{Introduction}

Risk pricing is a major issue for insurance companies for at least two obvious practical reasons: in the direct selling of insurance contracts to individual agents, companies have to compute and propose a premium to their clients and on the other hand, when insurance companies transfer a part of their underwritten risk through reinsurance, they also have to agree on a premium amount.

Several theoretical and practical premium calculations principle are available $[21,13,8]$, and the actuarial science literature includes systematic studies of these premium principles, in particular in relation with stochastic orders [16]. The indifference pricing, also called sometimes the zero utility principle asserts that the buyer's price should be such that the buyer is indifferent between paying nothing a not having a reinsurance (say) contract or paying the price now to receive reinsurance payments over suffered claims. "Indifferent" means here that her expected utility should be the same, with or without the contract, the price being the adjustment variable to achieve equality. Instead of expected utility, we consider in this paper the more practical point of view of monetary utility functions and convex risk measures (see Section 2.1 for precise definitions). Let us briefly describe our approach.

\subsection{Summary of the Approach}

When a reinsurance contract is purchased, it lowers the direct exposition of the insurance company, and potentially improves its wealth. Consequently, the needed regulatory capital is expected to decrease, which lowers the cost of immobilizing capital. In this paper, we measure the effect of the reinsurance contract on the ceding company's wealth using a monetary utility function $U$, and we measure the effect of capital immobilization using a convex or coherent risk measure $\rho$.

Let us assume that the firm supports a cost of capital $\bar{c}$, with $0<\bar{c}<1$. We denote $R$ the net result of the insurance company without a given reinsurance layer, and $R^{X L}(p)$ the net result with the layer, when paying a price $p$. We define the indifference premium, relative to the pair $(U, \rho)$, as the solution of the equation

$$
U[R-\bar{c} \rho(R)]=U\left[R^{X L}(p)-\bar{c} \rho\left(R^{X L}(p)\right)\right] .
$$

This price takes into account both the change in utility due to the layer addition and the change in reserves because of the cost of capital. When the reinsurance contract contains reinstatements, the total premium paid by the ceding company is a random variable (see Equation (2.5)), and we cannot directly apply the cash additivity property (Definitions 2.1 and 2.2) of $U$ and $\rho$ to compute $p$ explicitly. However, using both the convexity of $\rho$ and the concavity of $U$, it is possible to bound $p$ by two simple values, that are easily computable using simulations of the underlying risks. We also characterize the indifference price $p$ as the solution to a well-posed fixed point equation, which allows for numerical approximation of the price.

We provide numerical values of the indifference prices on a class of contracts with increasing limit and fixed capacity, on an underlying natural catastrophes risk corresponding to a real insurance portfolio (AXA). The shape of the obtained prices is comparable with indifference prices obtained from a set of simulated data. We include in our simulation study the case of Hawkes processes for claim arrival simulation.

The equation defining the indifference price can be written $\bar{U}(R)=\bar{U}\left(R^{X L}(p)\right)$, where $\bar{U}(X):=$ $U(X-\bar{c} \rho(X))$. The functional $\bar{U}$ can be seen a cash super-additive monetary utility function, in the sense that $\bar{U}(R+m) \geq \bar{U}(R)+m$, for each nonnegative constant value $m$. The random variables $R$ and $R^{X L}$ mentioned above are observed only at a terminal maturity time $t$, typically one year. To better understand the interpretation of the cash super-additivity inequality, note 
that $\bar{U}(1)=1+\bar{c}$, which says that the overall monetary utility of having 1 Euro of capital at time $t$ is equal to 1 plus the utility of the relief from having to hold capital, equal to $1 \times \bar{c}$. We discuss this further in Section 3.1.

\subsection{Related Literature}

The literature on reinsurance problems is large: we refer the reader to the monographs [1] and [8] and the references therein. Comparatively, there are little references concerning the pricing of reinsurance contracts containing reinstatements. Early references are [23], [24] and [3]. In particular, Sundt [24] provides a formula for the pure premium of contracts with reinstatements, which is adapted in [3] to the standard outputs of CAT models, known as event loss tables. The presence of reinstatements make the determination of the total aggregate losses distribution not obvious: several solutions are derived in [26] and [22]. This last reference also contains a comparison of the properties of different premium calculation principles, based on moments or on comonotonic risk measures. To avoid computing the probability law of the aggregate losses, Hürlimann [17] provides a distribution free approximation to pure premiums of layers with reinstatements, using maximal distributions for certain stochastic orders under moment constraints on the claim arrival process, and on the claim size distribution. The authors of [15] take an optimal contract point of view: the reinsurance contract parameters are chosen in order to minimize the expected distance between the loss and the premium income.

In a time dynamic setting, [25] and [26] study the ruin probability of a cedent having reinsurance with reinstatements, first with a pure premium assumption and then under standard deviation and PH-transform principles. The authors of [2] characterize the finite time ruin probability in a continuous time model, as the solution to a partial integro-differential equation and as the fixed point of a well behaved operator, which makes a numerical approximation possible.

The paper is organized as follows: in Section 2, we provide some introductory definitions and properties of risk measures and monetary utilities, then we precisely describe the class of reinsurance contracts we are interested in. We define the indifference price relative to both a monetary utility and a convex risk measure in Section 3, then we give some properties along with upper and lower bounds, for which we perform a simple asymptotic analysis. Section 4 contains numerical examples, from a real portfolio and from losses simulation.

\section{Model and Assumptions}

\subsection{Monetary Risk Measures and Utility Functions}

The indifference price considered in this paper is relative to both a monetary utility function and a risk measure. For clarity of the paper, we briefly provide here some definitions and intuitions, we refer the reader to [11] (Chapter 4) for a more complete treatment.

Risk Measures. Motivated by some imperfections of traditional risk measures such as the Value-at-Risk (VaR), which is recommended by the Solvency II European regulatory requirement for insurance companies, Artzner \& al. [4] and Frittelli and Rosazza-Gianin [12] introduced the notions of coherent and convex risk measures. These objects map a class $\mathcal{X}$ of random variables with $\mathbb{R}$. Typically, we take $\mathcal{X}=L^{\infty}(\mathbb{P})$ or $\mathcal{X}=L^{p}(\mathbb{P})$ with $p \geq 1$, where $(\Omega, \mathcal{F}, \mathbb{P})$ is a fixed probability space.

Definition 2.1. $\rho: \mathcal{X} \rightarrow \mathbb{R}$ is a law invariant coherent risk measure if

1. $\rho$ is monotone, in the sense that $X \leq Y$ almost surely implies that $\rho(X) \geq \rho(Y)$.

2. $\rho$ is cash-additive: $\forall m \in \mathbb{R}, \rho(X+m)=\rho(X)-m$. 
3. $\rho$ is convex : For each $X$ and $Y$ in $\mathcal{X}$ and for all $\lambda \in[0,1], \rho(\lambda X+(1-\lambda) Y) \leq \lambda \rho(X)+$ $(1-\lambda) \rho(Y)$.

4. $\rho$ is positively homogeneous: $\forall \lambda \in \mathbb{R}^{+}, \rho(\lambda X)=\lambda \rho(X)$.

5. $\rho$ is law-invariant: $X \stackrel{d}{=} Y$ implies that $\rho(X)=\rho(Y)$, where $\stackrel{d}{=}$ denotes equality in distribution.

If $\rho$ satisfies conditions 1 . to 4 . in Definition 2.1, then it is called a coherent risk measure, as introduced in [4]. We briefly discuss here the economic interpretations behind these properties. Let us start with property 2., which gives a unit to the value $\rho(X)$ : it says that $\rho(X)$ has the same unit as $m \in \mathbb{R}$. Since here $m \in \mathbb{R}$ is considered as a constant value of capital (Euros, Dollars etc.), then $\rho(X)$ also represents a capital amount. In particular, $\rho(X+\rho(X))=0$, so $\rho(X)$ is the constant amount of capital such that, when put in reserve in front of the risky position $X$, the new position $X+\rho(X)$ becomes riskless, from the point of view of $\rho$. Property 1 . is the most obvious: it states that if a revenue $Y$ is bigger than a revenue $X$ in all states of nature, we want to associate to it a lower risk value, and in particular a lower amount of reserve. The convexity property 3. corresponds to the idea that rational agents should prefer diversification, and under property 4., it is equivalent to the subadditivity of risks. The positive homogeneous property 4. says that $\rho$ is not sensitive to the size of positions, which is a strong assumption to make. On the other hand, it also says that the risk measurement procedure should be independent of the underlying currency, which would correspond to multiplication by a positive constant. Note that if $\rho$ is positively homogeneous, then $\rho$ is normalized, in the sense that $\rho(0)=0$. Finally, property 5 . is of a more probabilistic nature, and says that risk measurement only depends on the underlying distribution, and not on the specific origin of the risk.

Monetary Utility Functions. Risk measures are given a particular orientation: note that here positive random variables represent gains, so we consider non increasing risk measures, and cash-invariant with a minus sign. By opposition, a monetary utility is non decreasing, concave and cash-invariant with a plus sign.

Definition 2.2. $U: \mathcal{X} \rightarrow \mathbb{R}$ is a coherent monetary utility if

1. $U$ is monotone, in the sense that $X \leq Y$ almost surely implies that $U(X) \leq U(Y)$.

2. $U$ is cash-additive: $\forall m \in \mathbb{R}, U(X+m)=U(X)+m$.

3. $U$ is concave : For each $X$ and $Y$ in $\mathcal{X}$ and for all $\lambda \in[0,1], U(\lambda X+(1-\lambda) Y) \geq$ $\lambda U(X)+(1-\lambda) U(Y)$.

4. $U$ is positively homogeneous: $\forall \lambda \in \mathbb{R}^{+}, U(\lambda X)=\lambda U(X)$.

Consequently, if $\rho$ is a coherent risk measure, $U(X):=-\rho(X)$ defines a coherent monetary utility function. Except for the cash-invariance and positive homogeneity properties discussed above, the remaining properties of a monetary utility are standard assumptions in the economic literature. Note however that the class of monetary utility functions do not contain expected utilities as a particular case. On the contrary, the only expected utility function which is also cash-additive is linear, corresponding to a risk neutral agent. We refer the reader to the book [9] for a comprehensive treatment of monetary utility functions. Let us now give some examples of risk measures and monetary utilities that will be used in Section 4.

\section{Examples}

For a given random variable $X, \bar{F}_{X}$ denotes its survival function defined by $\bar{F}_{X}(x):=\mathbb{P}(X>x)$ and $\bar{q}_{X}$ denotes its tail quantile function, defined as the generalized inverse of $\bar{F}_{X}$ :

$$
\bar{q}_{X}(u):=\inf \left\{x \in \mathbb{R} \mid \bar{F}_{X}(x) \leq u\right\} .
$$


Distortion Risk Measures. Consider a distortion function $\psi:[0,1] \rightarrow[0,1]$, i.e. a non decreasing function with $\psi(0)=0$ and $\psi(1)=1$. Assuming that $\psi$ is left-continuous, we defined the distortion risk measure $\rho$ associated to $\psi$ by:

$$
\rho(X):=\int_{0}^{1} \bar{q}_{-X}(u) d \psi(u) .
$$

Such risk measures are monotone, cash-additive, positively homogeneous and law invariant. The only missing property to make them coherent is the convexity property 3. of Definition 2.1. If the probability space $(\Omega, \mathcal{F}, \mathbb{P})$ has no atoms, then one can prove that a risk measure of the form (2.1) is convex if and only if the distortion function $\psi$ is concave. Various examples of concave distortions are standard in the actuarial science literature (see eg. [27]), such as the proportional-hazard transform $\psi_{P H}(u):=u^{r}$, with $0<r<1$ (see Figure 6 and the associated example), or the Average Value-at-Risk (AVaR) distortion $\psi_{\alpha}(u):=\min \left(\frac{u}{\alpha}, 1\right)$ (which is the main example used in Section 4), which make these risk measures coherent.

Note that the class of distortion risk measures coincides with the class of comonotone risk measures. The next example provides a monetary utility (or equivalently, a risk measure) which is coherent, but not comonotone (see eg. [19], Section 3.2).

The Semi-deviation utility. For $0 \leq \delta \leq 1$ and $1 \leq p<+\infty$, define the semi-deviation utility as follows :

$$
U(X):=\mathbb{E}[X]-\delta \mathbb{E}\left[(X-\mathbb{E}(X))_{-}^{p}\right]^{1 / p}
$$

$U$ is a positively homogeneous law-invariant monetary utility function. $U$ favors the random variables with high expectation, and penalizes the (semi) deviations of $X$ away below its average $\mathbb{E}[X]$.

Dual Representation. Assume that $\mathcal{X}=L^{\infty}(\mathbb{P})$. A law invariant coherent monetary utility $U$ can be written as follows $[4,18]$ :

$$
U(X)=\inf _{Q \in \mathcal{Q}} E^{Q}[X], \quad X \in \mathcal{X},
$$

where $\mathcal{Q}$ denotes a set of admissible scenarios, which is a subset of the set $\mathcal{M}_{1}(\mathbb{P})$ of probability measures $Q$ that are absolutely continuous with respect to $\mathbb{P}$. This representation says that a coherent monetary utility is always a worst case expectation, over a set of possible scenarios. For instance, if $U(X)=-\int_{0}^{1} \bar{q}_{-X}(u) d \psi(u)$, where $\psi$ is a distortion function, then $\mathcal{Q}$ is the so-called core of $\psi \circ \mathbb{P}$, defined by

$$
\mathcal{Q}=\left\{Q \in \mathcal{M}_{1}(\mathbb{P}) \mid Q(A) \leq \psi(\mathbb{P}(A)), \text { for all } A \in \mathcal{F}\right\} .
$$

\subsection{Non Linear Contract with Reinstatements}

\section{The Contract Payoff}

Let $u_{0}$ be the initial surplus of an insurance company, and let $\beta$ be the gross premium income per time unit, so that the surplus process $R$ accumulated between times 0 and $t$, without reinsurance, is given by

$$
R(t)=u_{0}+\beta t-\sum_{i=1}^{N_{t}} X_{i}
$$

where $\left\{N_{t}, t \geq 0\right\}$ is a non decreasing integer valued pure jump process modelling the number of claims up to time $t$, and $\left\{X_{i}, i \in \mathbb{N}^{*}\right\}$ is a sequence of independent and identically distributed (i.i.d.) random variables modelling the individual claim sizes of the insurance portfolio. 
In an Excess-of-Loss (XL) reinsurance contract with retention $\ell$ and limit $m$, the reinsurer covers the part of each claim that exceeds $\ell$, up to the upper bound $\ell+m$. The reinsurer's part $Z_{i}$ and the insurer's part $C_{i}$ related to a claim $X_{i}$ are then given by

$$
\begin{aligned}
Z_{i} & =\left(X_{i}-\ell\right)^{+}-\left(X_{i}-\ell-m\right)^{+} \\
\text {and } \quad C_{i} & =\min \left(X_{i}, \ell\right)+\left(X_{i}-\ell-m\right)^{+} .
\end{aligned}
$$

Let

$$
Z(t):=\sum_{i=1}^{N_{t}} Z_{i}
$$

be the aggregate liability of the reinsurer. It can be limited to a maximal amount $M$. When $M$ is a multiple of the individual maximum cover $m$, i.e when $M=(k+1) m$, we say that the reinsurance contract contains $k$ reinstatements. In that case the aggregate liability of the reinsurer is given by $R_{k}(t):=\min (Z(t),(k+1) m)$. Each arrival of a new claim that consumes part of the layer is followed by the reconstitution of the layer cover, up to the remaining reinstatements.

For the $j$-th reinstatement, the cedent pays a premium $P_{j}$ given by

$$
P_{j}:=c_{j} p_{0} \frac{1}{m} \min [\max (Z(t)-(j-1) m, 0), m],
$$

where $p_{0}$ is the premium paid by the cedent at initiation of the contract, and where $c_{j}, j=1, \ldots, k$ are reinstatements premium, expressed as a percentage of $p_{0}$.

So the total premium paid by the cedent during the considered period is given by:

$$
\bar{P}(t):=p_{0}+\frac{1}{m} \sum_{j=1}^{k}\left(c_{j} p_{0}\right) \min [\max (Z(t)-(j-1) m, 0), m] .
$$

In other words, the payments are made following a pro-rata-capita principle: first, a premium $p_{0}$ is paid by the insurance company at initiation of the contract. Then, after arrival of each claim using up the layer cover, the cover is reinstated, in exchange of a premium payment $P_{j}$ given in (2.4). Note that $P_{j}$ is proportional to the fraction of the total layer capacity $m$ that is used by a claim. Note also that a new claim arrival which is greater than $\ell$, i.e. a jump of $Z(t)$, can either use up one reinstatement layer, or go through two reinstatements partly. For more clarity, we provide the Example 2.1 below, with detailed payments at each claim occurence. The wealth process of the cedent with reinsurance can be written as

$$
\begin{aligned}
R^{X L}(t) & =u_{0}+\beta t-\sum_{i=1}^{N_{t}} C_{i}-\bar{P}(t) \\
& =u_{0}+\beta t-\sum_{i=1}^{N_{t}} X_{i}+\sum_{i=1}^{N_{t}} Z_{i}-\bar{P}(t) \\
& =R(t)+\sum_{i=1}^{N_{t}} Z_{i}-\bar{P}(t)
\end{aligned}
$$

Remark 2.1. Note that the reinsurance contracts described above can include an aggregate deductible $L$ (see for instance [17]). In the present work, we take $L=0$ for simplicity, but all the results remain valid if $L>0$. Moreover, in the numerical results provided in Section 4.2, one can consider that the simulated distributions correspond to losses above L, instead of losses above 0 . 
In what follows, we will denote for simplicity

$$
\widetilde{N}(t):=\frac{1}{m} \sum_{j=1}^{k} c_{j} \min [\max (Z(t)-(j-1) m, 0), m],
$$

so that the random total payment writes $\bar{P}(t)=(1+\widetilde{N}(t)) p_{0}$. The random variable $\widetilde{N}(t)$, which lies between 0 and $k$, represents the number of used reinstatements, and does not have to be an integer.

Example 2.1. Let us consider a concrete example, allowing to better understand these contracts payoffs. Assume that the insurance company enters an XL contract with one year maturity, including two reinstatements and covering the losses between 200 and $300(\ell=200$ and $m=100)$. The initial premium required by the reinsurer is given by $p_{0}=10$. The costs of the first and second reinstatements correspond respectively to $80 \%$ and $50 \%$ of the initial premium $p_{0}\left(c_{1}=0.8\right.$ and $\left.c_{2}=0.5\right)$.

Assume that the first four losses are given by the following amounts:

1. $X_{1}=250$ : we have then $C_{1}=200$ and $Z_{1}=50$. The insurance company has to reinstate half of the layer, to do so it pays $50 * \frac{1}{100} * 80 \% * 10=4$.

If there were no more claims, the value of $\widetilde{N}(t)$ would be $80 \% * \frac{1}{100} * \min [\max (50,0), 100]=$ 0.4 .

2. $X_{2}=290$ : we have then $C_{2}=200$ and $Z_{2}=90$. The ceding company has to reinstate $90 \%$ of the layer. For this, it uses what is left of the first reinstatement (50) and a part of the second one (40). So it pays : $50 * \frac{1}{100} * 80 \% * 10+40 * \frac{1}{100} * 50 \% * 10=6$.

If there were no more claims, $\widetilde{N}(t)$ would be now equal to $0.4+50 * \frac{1}{100} * 80 \%+40 * \frac{1}{100} * 50 \%=$ 1.

3. $X_{3}=330$ : in that case all the layer has to be reinstated, but only $60 \%$ of the second reinstatement is available, the layer is then reinstated only up to this limit and the company pays: $60 * \frac{1}{100} * 50 \% * 10=3$.

All the reinstatements have been used and the value of $\widetilde{N}(t)$ is now $\sum_{j=1}^{k} c_{j}=1.3$.

4. $X_{4}=300$, the insurance company was only covered for the losses between 200 and 260, we have then here $Z_{4}=60$ and $C_{4}=240$. The contract stops and the ceding company is not reinsured for the rest of the year.

The total premium paid in this example amounts to $\bar{P}=10+4+6+3=23$. This value could have been lower if all the reinstatements had not been used before the end of the contract.

Reinstatement clauses allow the ceding insurance company to delay its premium payment in time, and to pay for new covers only when needed. In practice, it is usual that a pro-rata-temporis is also applied. For the reinsurer, the reinstatement clause has the obvious advantage to limit its total exposition.

\section{Indifference Pricing and Bounds}

The objective of this paper is the numerical approximation of the indifference price $p_{0}$ of a reinsurance layer with reinstatements, that we define as a solution of an equation indicating that the utility of an insurance company, when it buys the contract and pays the price $p_{0}$, is equal to its utility when it does not enter the transaction. Since the contract contains reinstatements, the total premium paid is random (see equation (2.5)). It is then not possible to use directly the cash-additivity property to calculate the indifference price. In the next sections we will bound this price by two easily computable values, when one has access to losses simulations. We will also show how to numerically compute $p_{0}$ as the fixed point of a well behaved function. 


\subsection{Cost of Capital and Cash Super-additivity}

Recall the definitions of $R$ and $R^{X L}$ given respectively in (2.3) and (2.6). For simplicity, we momentarily drop the dependence of $R$ and $R^{X L}$ on $t$ and insist on the fact that $R^{X L}\left(p_{0}\right)$ depends on $p_{0}$.

Assumption 3.1. The cedent maximizes its concave monetary utility function $U$, meaning that $U$ satisfies properties 1, 2 and 3 of Definition 2.2, and compute its needed regulatory capital using a convex risk measure $\rho$, i.e. satisfying properties 1, 2 and 3 of Definition 2.1. Moreover, $U$ and $\rho$ are normalized, in the sense that $U(0)=\rho(0)=0$.

Note that even if the class of concave monetary utilities coincides with the class of convex risk measures, we do not assume that $U$ and $\rho$ described in the previous assumption satisfy $U=-\rho$. Typical examples used in practice correspond to the coherent case, where both $U$ and $\rho$ are additionally positively homogeneous. Indeed, a common choice for $U$ in insurance and reinsurance practice is merely the expectation: the concave utility is just the expected return. Another common choice is the expected return, penalized by the standard deviation. A standard choice for a coherent $\rho$ in practice is the so-called Average Value-at-Risk defined in Section 2.1.

Definition 3.1. We say that $p_{0}$ is the indifference price of a given XL layer relatively to the pair $(U, \rho)$, if $p_{0}$ solves the equation

$$
U(R-\bar{c} \rho(R))=U\left(R^{X L}\left(p_{0}\right)-\bar{c} \rho\left(R^{X L}\left(p_{0}\right)\right)\right)
$$

where $\bar{c}$ is a given cost of capital.

This definition takes into account both the improvement of the total exposition due to the layer addition and the expected drop of the needed capital.

When the contract contains an unlimited number of free reinstatements (or when there are no reinstatements), then it is straightforward to determine the value of $p_{0}$, solution to (3.1). Indeed, in that case $\widetilde{N}(t)$ is equal to 0 and using the cash additivity properties of $U$ and $\rho,(3.1)$ can be rewritten

$$
U(R-\bar{c} \rho(R))=U\left(R(t)+\sum_{i=1}^{N_{t}} Z_{i}\right)-p_{0}-\bar{c} \rho\left(R(t)+\sum_{i=1}^{N_{t}} Z_{i}\right)-\bar{c} p_{0},
$$

which entails that

$$
p_{0}=\frac{\bar{U}\left(R(t)+\sum_{i=1}^{N_{t}} Z_{i}\right)-\bar{U}(R)}{1+\bar{c}},
$$

where $\bar{U}(X):=U(X-\bar{c} \rho(X))$. However, when the contract contains a clause of limited number of reinstatements, the random variable $\widetilde{N}(t)$ is not equal to 0 in general, and we cannot simply use the cash-additivity property to compute $p_{0}$.

Equation (3.1) can be written $\bar{U}(R)=\bar{U}\left(R^{X L}\left(p_{0}\right)\right)$. The functional $\bar{U}$ is cash super-additive, indeed:

$$
\bar{U}(R+m)=\bar{U}(R)+(1+\bar{c}) m \geq \bar{U}(R)+m, \quad \text { for } m \geq 0 .
$$

The cash additivity property has been largely discussed and criticized, since it requires that risky positions and reserve amounts are expressed in the same numéraire. However, insurance companies and financial institutions in general determine today the reserve amount to cover future risky positions. The cash-additivity property implicitly means that future risky amounts are discounted, using a deterministic discount factor. Otherwise, as discussed in [10], one should 
use cash-subadditive risk measures (or cash super-additive monetary utilities) since if $r$ denotes a deterministic interest rate, then $\rho(R-m)=\rho(R)+\frac{m}{1+r} \leq \rho(R)+m$, for $m \geq 0$.

Now we want to take into account the cost of collecting now an amount $m$ of capital, we would get $\rho(R-m)=\rho(R)+\frac{m}{1+r}(1+\bar{c})$. For simplicity, we will still assume that future risky incomes are already discounted, and we define $\bar{c}$ as the cost of raising capital, on top of the deterministic risk free interest rate. This is coherent with the fact that $\bar{U}(R+m)=\bar{U}(R)+(1+\bar{c}) m$. We refer to [10] for a much more detailed discussion and analysis of cash-additivity and its relaxations.

\subsection{A Fixed Point and Pricing Bounds}

We begin this subsection by proving that Equation (3.1) has a unique solution.

Proposition 3.1. If $U$ and $\rho$ are cash-additive, monotonic (respectively non-decreasing and nonincreasing), and respectively concave and convex, then there exists a unique $p_{0}$ solving Equation (3.1). Moreover, there exists a continuous nonincreasing function $\varphi$ such that $\varphi\left(p_{0}\right)=p_{0}$.

Proof. Again, we will drop here the dependence in $t$ of $R, R^{X L}$ and $\widetilde{N}$ for simplicity of notation. Recall that

$$
R^{X L}=R+\sum_{i=1}^{N_{t}} Z_{i}-(1+\tilde{N}) p_{0} .
$$

Using cash-additivity, we can write (3.1) as

$$
p_{0}=\frac{\bar{U}\left(R+\sum_{i=1}^{N_{t}} Z_{i}-p_{0} \tilde{N}\right)-\bar{U}(R)}{1+\bar{c}} .
$$

Now by concavity of $U$ and convexity of $\rho$, the map $p \mapsto \bar{U}\left(R+\sum_{i=1}^{N_{t}} Z_{i}-p \widetilde{N}\right)$ is concave, and thus continuous. By the assumed monotonicity properties, this map is also non-increasing. Let $\varphi(p):=\frac{\bar{U}\left(R+\sum_{i=1}^{N_{t}} Z_{i}-p \widetilde{N}\right)-\bar{U}(R)}{1+\bar{c}}$, so that $(3.3)$ is equivalent to $\varphi\left(p_{0}\right)=p_{0}$. We already know that $\varphi$ is continuous and non-increasing, and since $\varphi(0)>0, \varphi$ admits a unique fixed point. Note that the case where $\varphi(0)=0$ is excluded because it would imply that $Z_{i}=0$ almost surely, which has no interest in practice.

Note that in the above proposition and in its proof, we do not assume that $U$ or $\rho$ are positively homogeneous: this proposition covers the class of concave monetary utilities and convex risk measures, which are not necessarily coherent.

On top of proving existence and uniqueness of a solution to (3.1), we showed that the indifference price relative to the pair $(U, \rho)$ can be characterized as the unique fixed point of a monotonic function, which allows for a numerical approximation of $p_{0}$, that we carry out in Section 4.2.

We can also directly bound $p_{0}$ using both the concavity of $U$ and the convexity of $\rho$. To derive the bounds provided in Theorem 3.1 below, we need to introduce the following $\gamma$-dilatations.

For $U$ and $\rho$ satisfying Assumption 3.1, and for $\gamma>0$, we define

$$
\bar{U}_{\gamma}(X):=U_{\gamma}(X)-\bar{c} \rho_{\gamma}(X), \quad X \in \mathcal{X},
$$

where $U_{\gamma}$ and $\rho_{\gamma}$ are the $\gamma$-dilatations of $U$ and $\rho$, defined by

$$
U_{\gamma}(X):=\gamma U\left(\frac{1}{\gamma} X\right) \quad \text { and } \quad \rho_{\gamma}(X):=\gamma \rho\left(\frac{1}{\gamma} X\right) .
$$

By convexity of $\rho$ and concavity of $U$, one can prove that $\gamma \mapsto \rho_{\gamma}$ is non increasing and that $\gamma \mapsto U_{\gamma}$ is non decreasing (see Proposition 3.4 in [5]). Moreover,

$$
\bar{U}_{0}:=U_{0}-\bar{c} \rho_{0}:=\lim _{\gamma \rightarrow 0^{+}} U_{\gamma}-\bar{c} \lim _{\gamma \rightarrow 0^{+}} \rho_{\gamma}
$$


is well defined, where $\rho_{0}$ is the lowest coherent risk measure dominating $\rho$ and similarly, $U_{0}$ is the greatest coherent monetary utility dominated by $U$ (Proposition 3.8 in [5]). This criteria $\bar{U}_{0}$ naturally appears in the formulation of Theorem 3.1 below.

Lemma 3.1. Assume that $U$ is concave and $\rho$ is convex. Then for every $\lambda \in(0,1)$, and for every $X$ and $Y$ in $\mathcal{X}$,

$$
\rho(X+Y) \leq \rho_{\lambda}(X)+\rho_{1-\lambda}(Y) \quad \text { and } \quad \rho(X-Y) \geq \rho_{\frac{1}{\lambda}}(X)-\rho_{\frac{1-\lambda}{\lambda}}(Y)
$$

and we have by symmetry similar inequalities for $U$ :

$$
U(X+Y) \geq U_{\lambda}(X)+U_{1-\lambda}(Y) \quad \text { and } \quad U(X-Y) \leq U_{\frac{1}{\lambda}}(X)-U_{\frac{1-\lambda}{\lambda}}(Y) .
$$

Proof. We only prove the inequalities for $\rho$, that imply the ones for $U$ by replacing $\rho$ by $-\rho$. For the first inequality, note that by convexity of $\rho$, we get

$$
\begin{aligned}
\rho(X+Y) & =\rho\left(\lambda \frac{X}{\lambda}+(1-\lambda) \frac{Y}{1-\lambda}\right) \\
& \leq \lambda \rho\left(\frac{X}{\lambda}\right)+(1-\lambda) \rho\left(\frac{Y}{1-\lambda}\right) \\
& =\rho_{\lambda}(X)+\rho_{1-\lambda}(Y) .
\end{aligned}
$$

For the second inequality, we use the convexity of $\rho$ again to write

$$
\begin{aligned}
\rho(\lambda X) & =\rho\left(\lambda(X-Y)+(1-\lambda) \frac{\lambda}{1-\lambda} Y\right) \\
& \leq \lambda \rho(X-Y)+(1-\lambda) \rho\left(\frac{\lambda}{1-\lambda} Y\right),
\end{aligned}
$$

which entails that

$$
\begin{aligned}
\rho(X-Y) & \geq \frac{1}{\lambda} \rho(\lambda X)-\frac{1-\lambda}{\lambda} \rho\left(\frac{\lambda}{1-\lambda} Y\right) \\
& =\rho_{\frac{1}{\lambda}}(X)-\rho_{\frac{1-\lambda}{\lambda}}(Y) .
\end{aligned}
$$

We are now in a position to state the indifference price bounds, using the best coherent lower approximation $\bar{U}_{0}$ of $\bar{U}$, which plays a crucial role here.

Theorem 3.1. Assume that $U$ and $\rho$ satisfy the properties of Assumption 3.1. If $p_{0}$ is the indifference price of a given $X L$ layer with $k$ reinstatements relatively to the pair $(U, \rho)$, then

$$
p_{1} \leq p_{0} \leq p_{2},
$$

where

$$
p_{1}:=\frac{A}{1+\bar{c}-\bar{U}_{0}(-\widetilde{N}(t))}, \quad p_{2}:=\frac{A}{1+\bar{c}+\bar{U}_{0}(\widetilde{N}(t))}
$$

and

$$
A:=\bar{U}\left(R(t)+\sum_{i=1}^{N_{t}} Z_{i}\right)-\bar{U}(R(t))
$$

Remark 3.1. 1. From the definition of $p_{0}$ and the value of $A$ given above, it is clear that the indifference price $p_{0}$ and the associated bounds depend on what the contract buyer already has in portfolio. If there are running reinsurance contracts, then it would be straightforward to include them in $R(t)$. So the price depends here on how the considered additional layer diversifies the overall portfolio, which is a natural economic property. 
2. Note that $\widetilde{N}=0$, which corresponds to the case of an infinite number of free reinstatements leads to $p_{0}=p_{1}=p_{2}$ and these values coincide with the expression given in (3.2).

3. In the case where $\bar{c}=0$ and $U$ is given by the expectation, $p_{0}=p_{1}=p_{2}$ is equal to the pure premium, and the obtained formula matches the one given in [24].

4. From the expression (2.7), we see that $\widetilde{N}(t)$ is a sum of comonotone random variables, as non decreasing functions of $Z(t)$. If both $U$ and $\rho$ are comonotonic additive, which is the case if they are defined as integrals with respect to distorted probabilities as in $(2.1)$, then $\bar{U}_{0}=\bar{U}$ is also comonotonic additive and the expression $\bar{U}(\widetilde{N}(t))$ appearing in the upper bound $p_{2}$ can be simplified. Note however that $-\bar{U}(-\tilde{N}(t))$ does not simplify the same way, and this is the only thing differentiating the lower bound from the upper bound.

\section{Proof. of Theorem 3.1}

Equation (3.1) is equivalent to

$$
\begin{aligned}
& U\left(R(t)+\sum_{i=1}^{N_{t}} Z_{i}-p_{0}(1+\widetilde{N}(t))\right)-\bar{c} \rho\left(R(t)+\sum_{i=1}^{N_{t}} Z_{i}-p_{0}(1+\widetilde{N}(t))\right) \\
& =U(R(t))-\bar{c} \rho(R(t)) .
\end{aligned}
$$

Let $\lambda \in(0,1)$. Using Lemma 3.1 , the monotony properties of $\gamma \mapsto U_{\gamma}$ and $\gamma \mapsto \rho_{\gamma}$ and the fact that both $U_{0}$ and $\rho_{0}$ are coherent, we get

$$
\begin{aligned}
U\left(R(t)+\sum_{i=1}^{N_{t}} Z_{i}-p_{0}(1+\tilde{N}(t))\right) & \leq U_{\frac{1}{\lambda}}\left(R(t)+\sum_{i=1}^{N_{t}} Z_{i}\right)-U_{\frac{1-\lambda}{\lambda}}\left(p_{0}[1+\tilde{N}(t)]\right) \\
& \leq U_{\frac{1}{\lambda}}\left(R(t)+\sum_{i=1}^{N_{t}} Z_{i}\right)-p_{0} U_{0}(1+\tilde{N}(t))
\end{aligned}
$$

and

$$
\rho\left(R(t)+\sum_{i=1}^{N_{t}} Z_{i}-p_{0}(1+\widetilde{N}(t))\right) \geq \rho_{\frac{1}{\lambda}}\left(R(t)+\sum_{i=1}^{N_{t}} Z_{i}\right)-p_{0} \rho_{0}(1+\widetilde{N}(t)) .
$$

These inequalities, combined with (3.7) entail that

$$
p_{0} \bar{U}_{0}(1+\tilde{N}(t)) \leq \bar{U}_{\frac{1}{\lambda}}\left(R(t)+\sum_{i=1}^{N_{t}} Z_{i}\right)-\bar{U}(R(t)) .
$$

Now, since $1+\widetilde{N}(t)>0$ a.s. and since $U_{0}$ and $\rho_{0}$ are monotone and normalized (since $U$ and $\rho$ are), we get $\bar{U}_{0}(1+\widetilde{N}(t))>0$, from which we deduce the inequality

$$
p_{0} \leq \frac{\bar{U}_{\frac{1}{\lambda}}\left(R(t)+\sum_{i=1}^{N_{t}} Z_{i}\right)-\bar{U}(R(t))}{1+\bar{c}+\bar{U}_{0}(\widetilde{N}(t))} .
$$

This last inequality being verified for every $\lambda \in(0,1)$, we get

$$
p_{0} \leq \inf _{\lambda \in(0,1)}\left\{\frac{\bar{U}_{\frac{1}{\lambda}}\left(R(t)+\sum_{i=1}^{N_{t}} Z_{i}\right)-\bar{U}(R(t))}{1+\bar{c}+\bar{U}_{0}(\widetilde{N}(t))}\right\}=\frac{\bar{U}_{1}\left(R(t)+\sum_{i=1}^{N_{t}} Z_{i}\right)-\bar{U}(R(t))}{1+\bar{c}+\bar{U}_{0}(\widetilde{N}(t))}=p_{2} .
$$

since $\gamma \mapsto \bar{U}_{\gamma}$ is non decreasing, which completes the proof of the upper bound. 
For the lower bound, using Lemma 3.1 again, we get

$$
\begin{aligned}
U\left(R(t)+\sum_{i=1}^{N_{t}} Z_{i}-p_{0}(1+\widetilde{N}(t))\right) & \geq U_{\lambda}\left(R(t)+\sum_{i=1}^{N_{t}} Z_{i}\right)+p_{0} U_{0}(-1-\widetilde{N}(t)) \text { and } \\
\rho\left(R(t)+\sum_{i=1}^{N_{t}} Z_{i}-p_{0}(1+\widetilde{N}(t))\right) & \leq \rho_{\lambda}\left(R(t)+\sum_{i=1}^{N_{t}} Z_{i}\right)+p_{0} \rho_{0}(-1-\widetilde{N}(t)) .
\end{aligned}
$$

By plugging these inequalities in (3.7), we have

$$
p_{0} \geq \frac{\bar{U}_{\lambda}\left(R(t)+\sum_{i=1}^{N_{t}} Z_{i}\right)-\bar{U}(R(t))}{1+\bar{c}-\bar{U}_{0}(-\widetilde{N}(t))},
$$

where we have used that $1+\bar{c}-\bar{U}_{0}(-\tilde{N}(t))=\bar{U}_{0}(-1-\tilde{N}(t))<0$, since $U_{0}$ and $\rho_{0}$ are monotone and normalized. The last inequality being satisfied for every $\lambda \in(0,1)$, we have

$$
p_{0} \geq \sup _{\lambda \in(0,1)}\left\{\frac{\bar{U}_{\lambda}\left(R(t)+\sum_{i=1}^{N_{t}} Z_{i}\right)-\bar{U}(R(t))}{1+\bar{c}-\bar{U}_{0}(-\widetilde{N}(t))}\right\}=p_{1},
$$

since $\lambda \mapsto \bar{U}_{\lambda}$ is non decreasing.

Remark 3.2. The monotony properties of $U$ and $\rho$ implies that $0 \leq p_{1}$. Furthermore, the concavity and convexity properties of $U$ and $\rho$ implies that we have indeed $p_{1} \leq p_{2}$.

Let us provide an example, illustrating in particular the value $U_{0}$. Assume that $\mathcal{X}=L^{\infty}(\mathbb{P})$. The main example of concave but not coherent monetary functional is probably the entropic monetary utility, defined by

$$
U(X):=-\log \mathbb{E}[\exp (-X)], \quad X \in \mathcal{X} .
$$

The $\gamma$-dilatation of $U$ has the following well known dual representation

$$
U_{\gamma}(X):=-\gamma \log \mathbb{E}\left[\exp \left(-\frac{1}{\gamma} X\right)\right]=\inf _{Q \in \mathcal{M}_{1}(\mathbb{P})}\left\{E^{Q}(X)-\gamma H(Q, \mathbb{P})\right\}
$$

where $H(Q, \mathbb{P})$ denotes the relative entropy of $Q$ with respect to the reference probability measure $\mathbb{P}$ and where $\mathcal{M}_{1}(\mathbb{P})$ is the set of probability measures on $(\Omega, \mathcal{F})$ which are absolutely continuous with respect to $\mathbb{P}$. When $\gamma$ goes to 0 , the $\gamma$-dilatation $U_{\gamma}$ converges to the most conservative coherent monetary utility $U_{0}$ given by

$$
U_{0}(X)=\inf _{Q \in \mathcal{M}_{1}(\mathbb{P})}\left\{E^{Q}(X), H(Q, \mathbb{P})<+\infty\right\}=\mathbb{P}-\operatorname{ess} \inf (X) .
$$

Assume for simplicity that $\rho$ is coherent, so that $\rho_{\gamma}=\rho$. In that case, Theorem 3.1 says that

$$
\frac{\bar{U}\left(R(t)+\sum_{i=1}^{N_{t}} Z_{i}\right)-\bar{U}(R(t))}{1+\bar{c}+k+\bar{c} \rho(\widetilde{N}(t))} \leq p_{0} \leq \frac{\bar{U}\left(R(t)+\sum_{i=1}^{N_{t}} Z_{i}\right)-\bar{U}(R(t))}{1+\bar{c}-\bar{c} \rho(\widetilde{N}(t))} .
$$

since

$$
\begin{aligned}
\bar{U}_{0}(\tilde{N}(t)) & =\operatorname{ess} \inf (\widetilde{N}(t))-\bar{c} \rho(\widetilde{N}(t)) \\
& =-\bar{c} \rho(\widetilde{N}(t)) \quad \text { and } \\
-\bar{U}_{0}(-\widetilde{N}(t)) & =-\operatorname{ess} \inf (-\widetilde{N}(t))+\bar{c} \rho(\widetilde{N}(t)) \\
& =k+\bar{c} \rho(\widetilde{N}(t)) .
\end{aligned}
$$


When both $U$ and $\rho$ are additionally positively homogeneous, then these measures are of course invariant under $\gamma$-dilatation and in particular, $\bar{U}_{0}=\bar{U}$.

Corollary 3.1. Let $U$ be a coherent monetary utility and $\rho$ be a coherent risk measure. Then

$$
\frac{\bar{U}\left(R(t)+\sum_{i=1}^{N_{t}} Z_{i}\right)-\bar{U}(R(t))}{1+\bar{c}-\bar{U}(-\tilde{N}(t))} \leq p_{0} \leq \frac{\bar{U}\left(R(t)+\sum_{i=1}^{N_{t}} Z_{i}\right)-\bar{U}(R(t))}{1+\bar{c}+\bar{U}(\tilde{N}(t))} .
$$

In the next section, we numerically compute these bounds in the case of a real insurance portfolio, and analyze them using simulated losses.

\subsection{Asymptotic Analysis}

The bounds obtained in the previous theorem are useful when for high layers, i.e. layers with high retention, which is clearly visible from the numerical results presented in the next section. To make this more precise, we provide here some asymptotic results in the regime where the retention $\ell$ goes to infinity. Interestingly, in the case where $\mathcal{X}=L^{\infty}(\mathbb{P})$, the needed continuity properties to be able to take a limit are derived from the cash-additivity properties of $U$ and $\rho$. So we will assume in this subsection that $\mathcal{X}=L^{\infty}(\mathbb{P})$, to be able to use the fact that cashadditive functionals are continuous with respect to the uniform norm. If $\mathcal{X}=L^{p}(\mathbb{P})$, with $p \geq 1$ it is the concavity and convexity of $U$ and $\rho$ that entail continuity, and the same analysis can be carried out using the results from [6].

Proposition 3.2. Assume that $U$ and $\rho$ satisfy the properties of Assumption 3.1 and let $p_{0}(\ell)$, $p_{1}(\ell)$ and $p_{2}(\ell)$ the indifference price and the bounds given in Theorem 3.1, expressed as functions of $\ell$, for a fixed $m>0$. We have

$$
\left|p_{2}(\ell)-p_{1}(\ell)\right| \rightarrow 0 \text { when } \ell \text { goes to infinity }
$$

Proof. Recall that

$$
A(\ell):=A=\bar{U}\left(R(t)+\sum_{i=1}^{N_{t}} Z_{i}\right)-\bar{U}(R(t)), \quad \text { with } \quad Z_{i}=\left(X_{i}-\ell\right)^{+}-\left(X_{i}-\ell-m\right)^{+} .
$$

$\bar{U}$ is continuous with respect to the uniform norm of $L^{\infty}(\mathbb{P})$. To see this, notice first that $X \leq Y+\|X-Y\|_{\infty}$ for any $X$ and $Y$ in $L^{\infty}(\mathbb{P})$. The monotonicity and cash additivity of $U$ and $\rho$ give,

$$
\bar{U}(X) \leq \bar{U}(Y)+(1+\bar{c})\|X-Y\|_{\infty} .
$$

Reversing the role played by $X$ and $Y$, we obtain the desired continuity. It is clear that $\sum_{i=1}^{N_{t}} Z_{i}$ converges almost surely to 0 , which implies that it converges also in $L^{\infty}(\mathbb{P})$ norm. This implies that $A(\ell)$ converges to 0 when $\ell$ goes to infinity.

By noticing that $\widetilde{N}(t)$ also converges almost surely and hence in $L^{\infty}(\mathbb{P})$ to 0 when $\ell$ goes to $+\infty$, we get that $-\bar{U}_{0}(-1-\tilde{N}(t)) \rightarrow-\bar{U}_{0}(-1)=1+\bar{c}$ and $\bar{U}_{0}(1+\tilde{N}(t)) \rightarrow \bar{U}_{0}(1)=1+\bar{c}$. This entails that $\left|p_{2}(\ell)-p_{1}(\ell)\right|=p_{2}(\ell)-p_{1}(\ell) \rightarrow 0$.

Remark 3.3. The previous proposition also says that $p_{0}(\ell)$, the unique solution to (3.1) converges to 0 , which is obvious from an economic standpoint: the indifference price of a layer with reinstatements and arbitrarily large retention should be arbitrarily close to 0 . 


\section{Numerical Analysis and Examples}

\subsection{Results for a Real Insurance Portfolio}

In this subsection, we describe the values obtained for the bounds $p_{1}$ and $p_{2}$, that were computed using an internal natural catastrophe model developed by the french insurer AXA. This model took as input the risk characteristics of a European portfolio, and provided simulated losses as output. For confidentiality reasons, we will not be more specific about this portfolio. However, for comparison and reproducibility purposes, we also do the same exercice with simulated losses in the next subsection, and provide the developed $\mathrm{R}$ code as supplementary material to this article.

In figure 1 below, we represented the upper and lower bounds $p_{1}$ and $p_{2}$ obtained for layers with retention equal to $m+\ell=10^{8}(n+1)$ euros and limit equal to $m=10^{8}$ euros, for $n$ varying from 0 to 32. In other words, we computed the bound prices for 33 layer contracts, with the same limit value, for a retention ranging from one hundred million euros to 3.3 billion euros. All the contracts contain $k=4$ possible reconstitutions, and each reconstitution when it happens, is paid at $c=100 \%$ of the initial price. The prices are expressed as a percentage of $m=10^{8}$, which correspond to rates on line (RoL) in the reinsurance terminology. Note that for confidentiality reasons, we have applied a a constant shift to the obtained prices, which does not change the shape of the curves.

These reinsurance contracts cover an insurance portfolio of risk of natural catastrophes type. The calculations have been made using 25000 years of simulations of losses from the considered portfolio.

From expression (2.1), we get that the Average Value-at-Risk can be written $\rho(X)=\frac{1}{\alpha} \int_{0}^{\alpha} q_{-X}(u) d u$ and recall the expression (2.2) of the semi-deviation monetary utility function: the monetary utility used here is the semi-deviation utility with parameters $p=2$ and $\delta=\frac{1}{2}$. The coherent risk measure is the $A V a R_{\alpha}$ with $\alpha=1 / 200$.

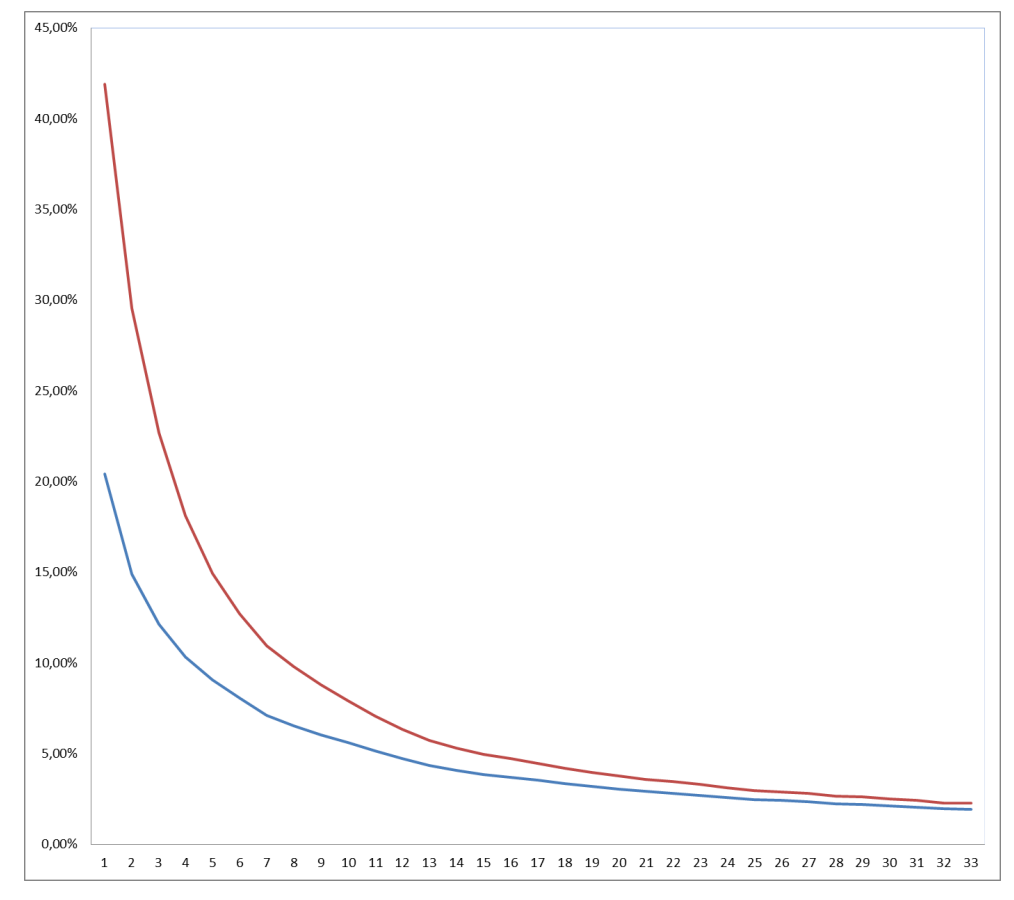

Figure 1: Rate on Line (RoL) bounds for a catastrophe insurance portfolio

We can see that the gap between $p_{1}$ and $p_{2}$ is important for the first contracts. This is due to the fact that the first contracts correspond to intermediate retentions, for which the variance of the random variable $\widetilde{N}$ is large. In that case, the different quantities $-\bar{U}(-\widetilde{N})$ and $U(\widetilde{N})$ contribute 
considerably to the gap between $p_{1}$ and $p_{2}$.

However, for contracts with a high retention, beginning from 1.3 billion Euros $(n=12)$, the bounds are acceptable in practice. For intermediate retentions, where the bounds usefulness is limited, it is still possible to compute $p_{0}$ efficiently. This what we do next relying on simulated losses.

\subsection{Simulated Losses and Hawkes Processes}

Hawkes processes [14] form a class of counting processes where current values are influenced by past events, in particular by the past number of jumps: this is the so-called self-exciting property. More precisely, the inhomogeneous intensity of a Hawkes process $\left(N_{t}\right)_{t \geq 0}$ is expressed as

$$
\lambda_{t}=\lambda_{0}+\sum_{T_{n}<t} \phi\left(t-T_{n}\right)
$$

where $\left(T_{n}\right)_{n \geq 1}$ are the jump times of $N, \lambda_{0}>0$ and $\phi$ is a nonnegative function converging to 0 when $t$ goes to infinity. So if a jump occurs at time $T_{n}$, the intensity increases by $\phi\left(t-T_{n}\right)$ : this models the self-exciting property. On the other hand, since $\phi$ returns to 0 after a large time, the self-excitation tends to disappear.

In [14], Hawkes considers the particular case of exponential decay: $\phi(t)=\alpha_{h} \exp \left(-\beta_{h} t\right)$, with $\alpha_{h}>0$ and $\beta_{h}>0$. In that case, each jump arrival instantaneously increases the intensity by $\alpha_{h}$, then this influence decreases over time at rate $\beta_{h}$. Note that when $\alpha_{h}=0, \lambda_{t}$ is constant equal to $\lambda_{0}$ and $N$ is merely a Poisson process with intensity $\lambda_{0}$.

In Figure 2, we depicted the obtained indifference price and associated upper and lower bounds of various reinsurance layers, with fixed capacity $m=2$ and retention ranging from $\ell=10$ to $\ell=20$. To generate this figure, we simulated $10^{5}$ trajectories of losses, where claim arrivals are driven by a Poisson process with intensity $\lambda_{0}=1$ and claim sizes are given by independent Pareto random variables with threshold parameter equal to 6 and shape parameter equal to 4 . The monetary utility is given by the semi-deviation utility with $p=2$ and $\delta=0.5$ and the risk measure is the Average Value-at-Risk with parameter $\alpha=0.2$. The cost of capital over the risk free rate amounts to $3 \%$. The underlying reinsurance contract contains $k=2$ reinstatements, with prices $c_{1}=c_{2}=1$. The bounds are again not very good for small retentions, where the price is unrealistically high anyway, since for those levels of retention the cover is systematically used. The bounds are acceptable for intermediate and high retentions. 


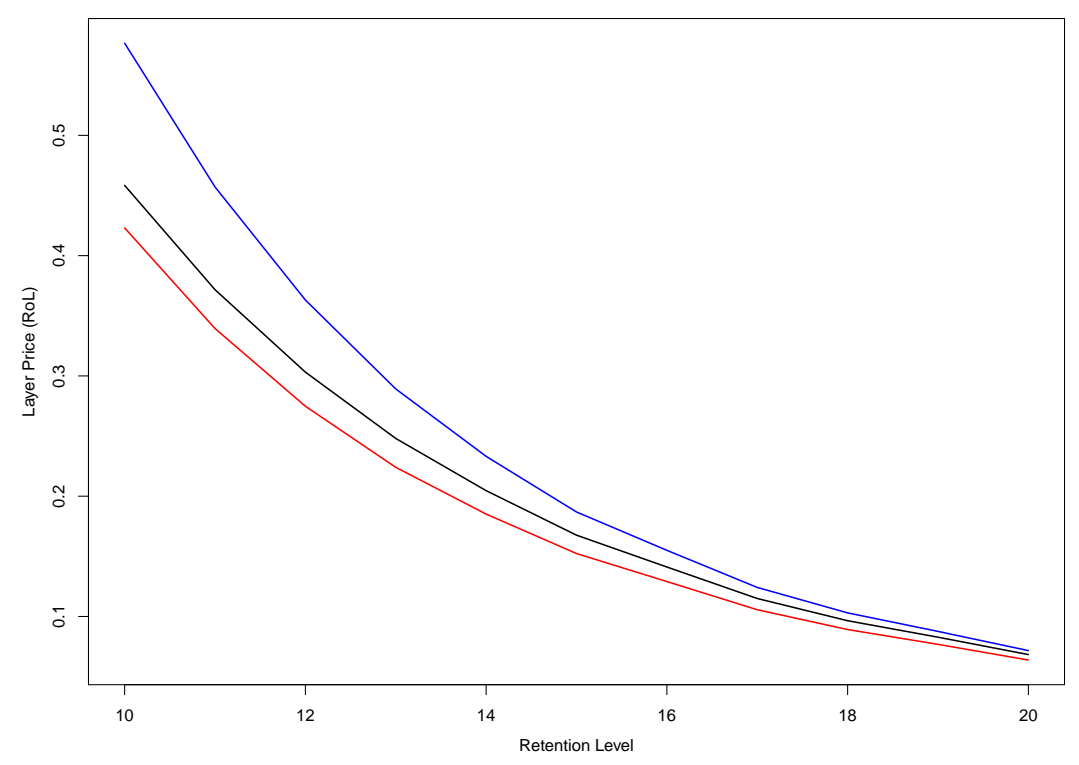

Figure 2: Rate on Line (RoL) bounds - Simulated data - Poisson case

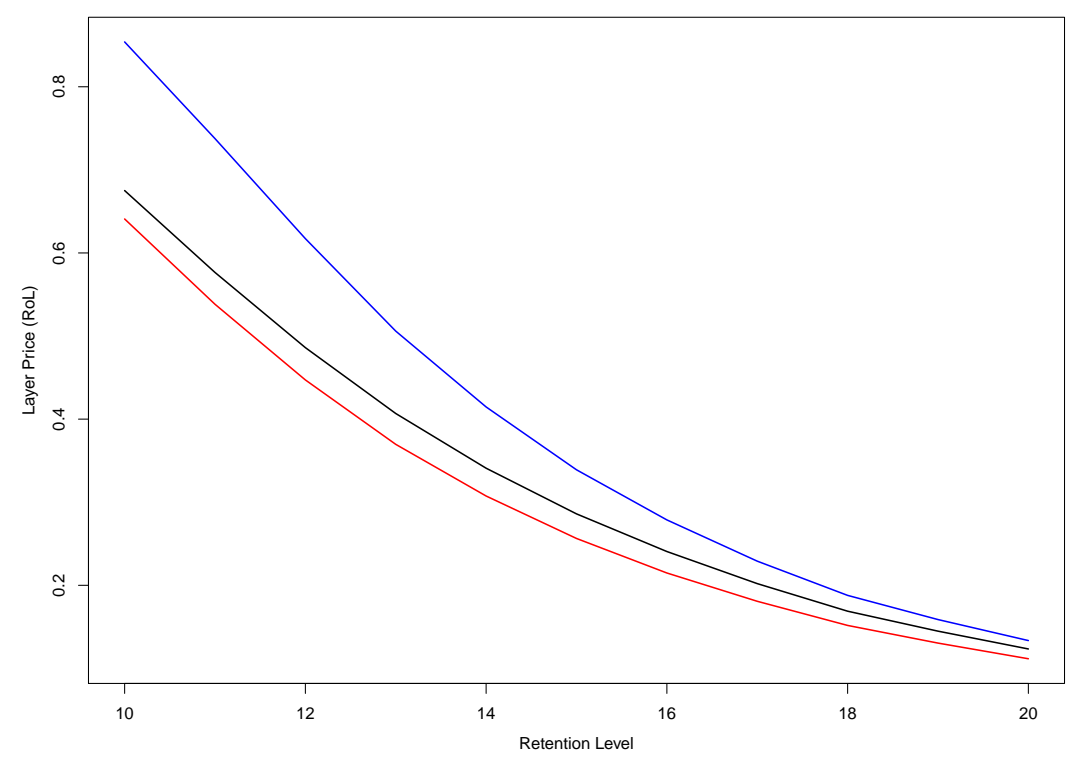

Figure 3: Rate on Line (RoL) bounds - Simulated data - Hawkes process case

To have an idea of the effect of dependence among claim arrivals, we did the same exercice with a Hawkes process, with parameters $\alpha_{h}=0.5, \beta_{h}=0.7$ and the base intensity remains equal to $\lambda_{0}=1$. All the other parameters are kept unchanged. Figure 3 shows the results on the same class of reinsurance contracts with fixed capacity and varying retention. Not surprisingly, the effect of introducing a self-excitation feature on claim arrivals leads to a substantial price increase.

To further illustrate the price increase, we fixed a particular layer: $\ell=18$ and $m=2$. We represented in Figure 4 the indifference price and bounds for this layer, where the self-excitation parameter ranges between $\alpha_{h}=0.05$ and $\alpha_{h}=\beta_{h}-0.05=0.65$. This confirms the non negligible effect on the price, that more than doubles in our particular case. 


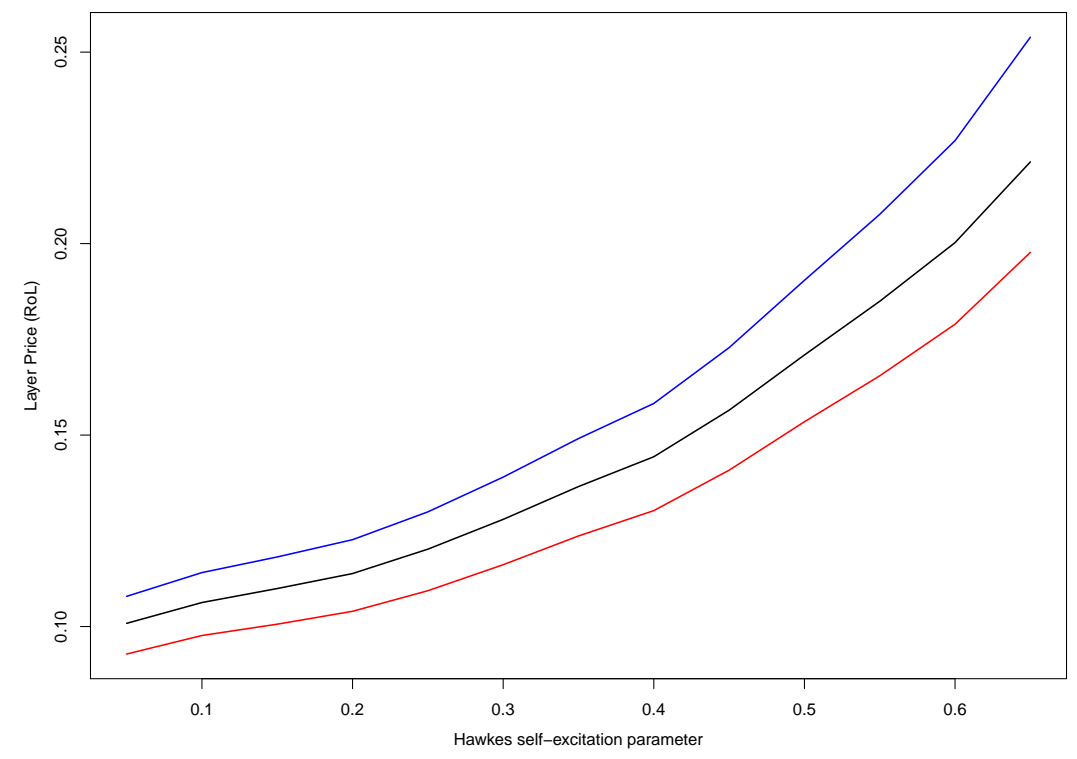

Figure 4: RoL of the 2XS18 contract, as a function of the self-excitation parameter

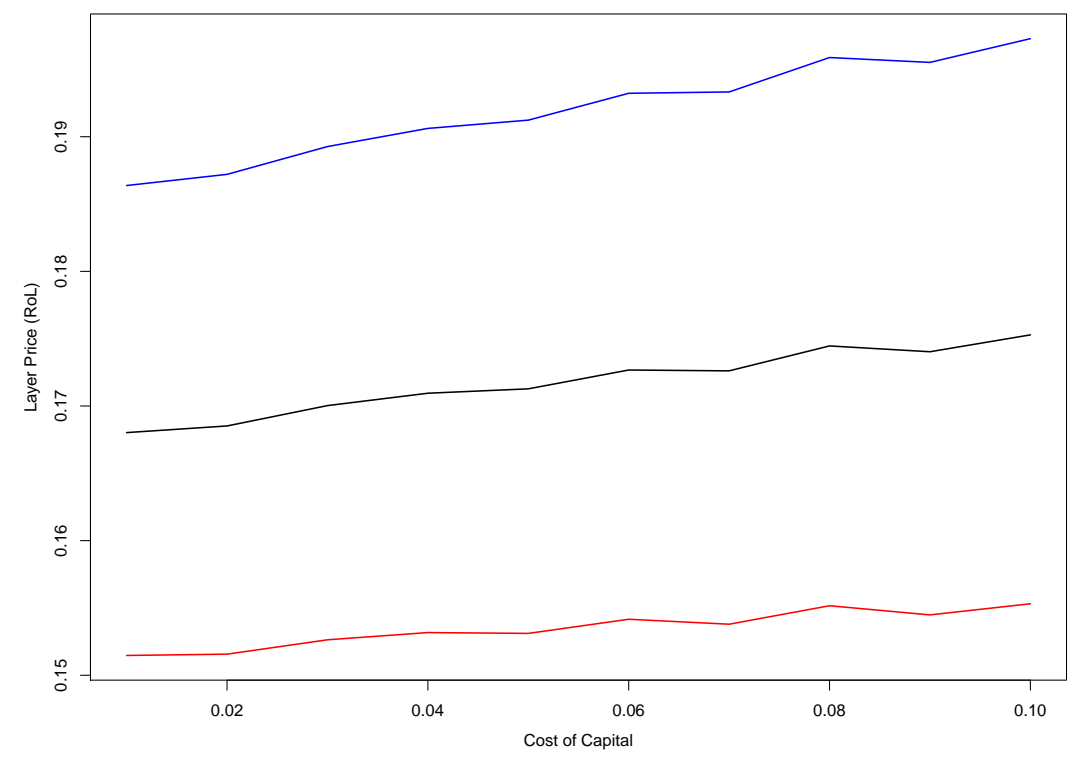

Figure 5: RoL of the 2XS18 contract, as a function of the cost of capital

As already mentioned, the cost of capital value taken to generate this graphs, which represents the cost over the risk free rate, is $\bar{c}=3 \%$. This is suggested by both the empirical analysis conducted by A. Damodaran [7], available online and the annual cost of capital study [20] developed by a consulting firm, and also available online. In Figure 5, we also considered the single contract with $\ell=18$ and $m=2$, with varying cost of capital parameter, from $\bar{c}=1 \%$ to $\bar{c}=10 \%$. We observe that the effect on the indifference price is not significant.

To end this section, let us illustrate the impact on the bounds and on the price of a change in the utility function. This analysis readily explains a change in the risk measure, that we keep fixed for simplicity, and insist rather on the utility, that has a greater impact here, due to the term $\bar{c}$ in front of the risk measure. Figure 6 shows the price and bound values for different coherent 
monetary utilities, given by :

$$
U_{r}(X)=-\int_{0}^{1} \bar{q}_{-X}(u) d \psi_{r}(u)
$$

where $\psi_{r}(u):=u^{r}$ with $r \in(0,1] . U_{r}$ is the so-called Proportional Hazard $(\mathrm{PH})$ transform with parameter $r$. For $r=1, U_{r}$ is merely the expectation, and as $r$ approaches $0, \psi_{r}$ very quickly takes values close to one, and $U_{r}$ approaches the worst case monetary utility. So $r$ close to 0 corresponds to a very risk averse agent, ready to accept high prices for a reinsurance cover, and when $r$ approaches $1, U_{r}$ corresponds to a risk neutral agent. The risk measure chosen to generate Figure 6 is the expectation $\rho(X)=-E^{\mathbb{P}}[X]$. When $r=1$, both $U_{r}$ and $\rho$ are linear functionals, and one can check then that the bounds from Theorem 3.1 coincide, which is clear in Figure 6. On the other hand, when $r$ is close to 0 , the distance between the bounds is large and prices are larger.

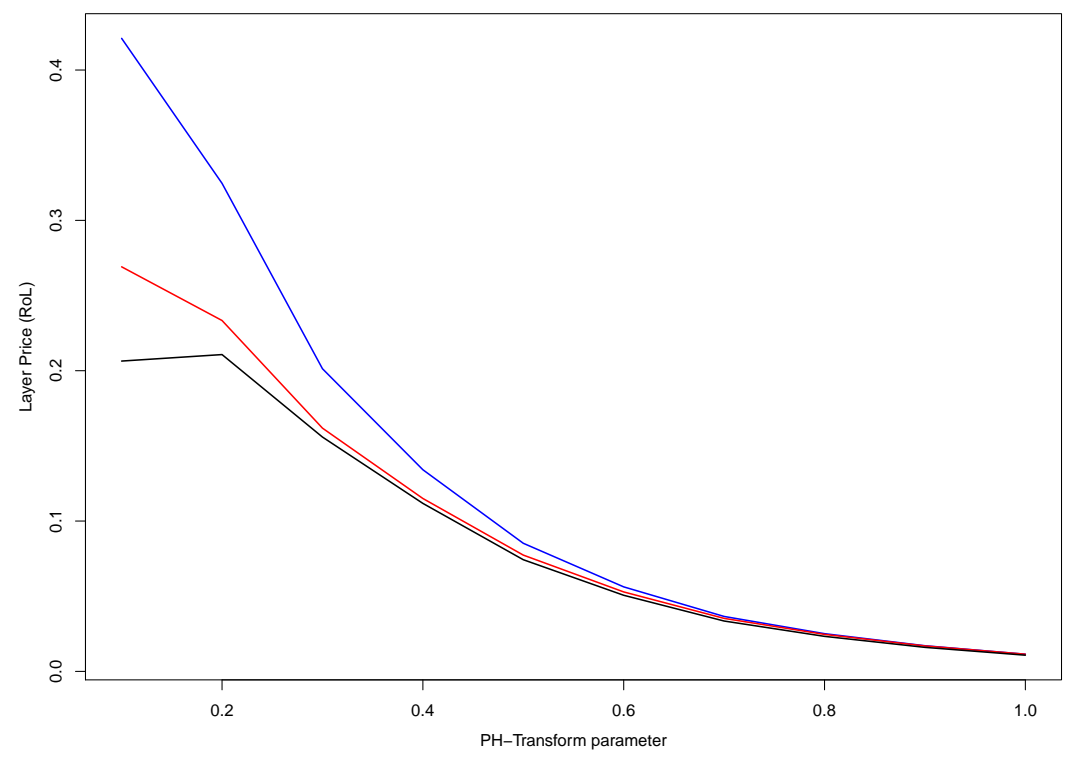

Figure 6: RoL of the 2XS18 contract, as a function of PH-transform parameter

The $R$ code to generate the simulations and all the figures is made available as a supplementary material to this article, for comparison and reproducibility.

\section{Conclusion}

The approach we adopted in this paper allowed us to study indifference prices of reinsurance layers with reinstatements, both theoretically and from an applied and numerical perspective, via a simulation study, including the non standard case of dependence between claim arrivals. We exploited the convexity and cash additivity properties of coherent monetary criteria to analyze lower and upper bounds on the considered indifference price.

We only considered here a static point of view. It would be natural to extend this simple model to a time dynamic framework where investment in financial markets is allowed for both the insurer and the reinsurer, given that the reinsurer can choose the parameters of a contract with reinstatements: this would lead to a mixed optimal contracting and indifference pricing stochastic control problem, that we leave for further research. 


\section{References}

[1] Albrecher, H., Beirlant, J., And Teugels, J. L. Reinsurance: actuarial and statistical aspects. John Wiley \& Sons, 2017.

[2] Albrecher, H., And HaAs, S. Ruin theory with excess of loss reinsurance and reinstatements. Applied mathematics and computation 217, 20 (2011), 8031-8043.

[3] Anderson, R. R., And Dong, W. Pricing catastrophe reinsurance with reinstatement provisions using a catastrophe model. In Casualty Actuarial Society Forum (1998), pp. 303322.

[4] Artzner, P., Delbaen, F., Eber, J.-M., and Heath, D. Coherent measures of risk. Mathematical finance 9, 3 (1999), 203-228.

[5] Barrieu, P., and El Karoui, N. Pricing, hedging, and designing derivatives with risk measures., indifference pricing: Theory and applications, 2009.

[6] Biagini, S., And Frittelli, M. On the extension of the namioka-klee theorem and on the fatou property for risk measures. In Optimality and risk-modern trends in mathematical finance. Springer, 2009, pp. 1-28.

[7] Damodaran, A. Cost of capital by sector. http://people.stern.nyu.edu/adamodar/ New_Home_Page/datafile/wacc.htm. Accessed: 2020-06-05.

[8] Deelstra, G., and Plantin, G. Risk theory and reinsurance. Springer, 2014.

[9] Delbaen, F. Monetary utility functions. Osaka University Press, Osaka, 2012.

[10] El Karoui, N., And Ravanelli, C. Cash subadditive risk measures and interest rate ambiguity. Mathematical Finance: An International Journal of Mathematics, Statistics and Financial Economics 19, 4 (2009), 561-590.

[11] Föllmer, H., AND Schied, A. Stochastic finance: an introduction in discrete time. Walter de Gruyter, 2011.

[12] Frittelli, M., And Gianin, E. R. Putting order in risk measures. Journal of Banking E Finance 26, 7 (2002), 1473-1486.

[13] Goovaerts, M. J., De Vylder, F., And Haezendonck, J. Insurance premiums: theory and applications. North-Holland Amsterdam, 1984.

[14] Hawkes, A. G. Spectra of some self-exciting and mutually exciting point processes. Biometrika 58, 1 (1971), 83-90.

[15] Hess, K. T., And Schmidt, K. D. Optimal premium plans for reinsurance with reinstatements. ASTIN Bulletin: The Journal of the IAA 34, 2 (2004), 299-313.

[16] Hürlimann, W. On stop-loss order and the distortion pricing principle. ASTIN Bulletin: The Journal of the IAA 28, 1 (1998), 119-134.

[17] Hürlimann, W. Excess of loss reinsurance with reinstatements revisited. ASTIN Bulletin: The Journal of the IAA 35, 1 (2005), 211-238.

[18] Jouini, E., Schachermayer, W., And Touzi, N. Law invariant risk measures have the fatou property. In Advances in mathematical economics. Springer, 2006, pp. 49-71. 
[19] Jouini, E., Schachermayer, W., and Touzi, N. Optimal risk sharing for law invariant monetary utility functions. Mathematical Finance: An International Journal of Mathematics, Statistics and Financial Economics 18, 2 (2008), 269-292.

[20] KPMG. Cost of capital study 2019. https://home.kpmg/de/en/home/insights/2019/ 10/cost-of-capital-study-2019.html. Accessed: 2020-06-05.

[21] Laeven, R. J., And Goovaerts, M. J. Premium Calculation and Insurance Pricing. 2014.

[22] Mata, A. J. Pricing excess of loss reinsurance with reinstatements. ASTIN Bulletin: The Journal of the IAA 30, 2 (2000), 349-368.

[23] Rytganad, M. On calculating the risk premium for an excess of loss cover with an annual aggregate deductible and a limited number of reinstatements. Astin Colloquium, Copenhagen, Denmark 1, 27 (1996), 82-94.

[24] Sundt, B. On excess of loss reinsurance with reinstatements. Insurance: Mathematics and Economics 12, 1 (1993), 73.

[25] Walhin, J.-F., And PARIS, J. The effect of excess of loss reinsurance with reinstatements on the cedent?s portfolio. Blätter der Deutschen Gesellschaft für Versicherungsmathematik 24 (2000), 616-627.

[26] Walhin, J.-F., AND PARIS, J. Excess of loss reinsurance with reinstatements: premium calculation and ruin probability of the cedent. Blätter der DGVFM 25, 1 (2001), 1-12.

[27] WANG, S. Premium calculation by transforming the layer premium density. ASTIN Bulletin: The Journal of the IAA 26, 1 (1996), 71-92. 